\title{
MICROCULTURAS Y CULTURA ORGANIZACIONAL: CONSTRUCCIÓN DIALÉCTICA EN LA ORGANIZACIÓN*
}

\author{
DAIMER HIGUITA LÓPEZ** \& JULIETH C. LEAL GARCÍA*** \\ UNIVERSIDAD NACIONAL DE COLOMBIA
}

Recibido/ Received/Recebido: 22/09/2009 - Aceptado/ Accepted/Aprovado: 15/01/2010

\begin{abstract}
Resumen
Las disciplinas administrativas han construido un modelo conceptual de cultura organizacional en donde se ha desconocido el carácter natural que le concede la antropología al concepto de cultura. La investigación buscó indagar por la manera como la estructura formal de organizaciones productivas incide en la cooperación espontánea de los empleados. El artículo tiene como propósito evidenciar que la cultura en la organización no solo se configura por los aspectos de la estructura formal sino que existen otras dinámicas grupales espontáneas, distintas y a la vez, complementarias de los postulados que maneja la gestión de la cultura organizacional. El trabajo se apoya en algunos conceptos claves como orden, vinculación y principios de organización social, que permiten presentar un paralelo entre dos tipos de orden: el orden espontáneo y el orden artificial. Finalmente, se devela la forma en cómo el orden artificial restringe, en algunos casos, las interacciones sociales entre los empleados.
\end{abstract}

Palabras clave: cultura, cultura organizacional, orden social, vínculo social, grupo formal, grupo informal

\section{MICROCULTURES AND ORGANIZATIONAL CULTURE: DIALECTIC CONSTRUCTION IN THE ORGANIZATION}

\begin{abstract}
Administrative disciplines have built a conceptual model of organizational culture, where the natural character that culture concept enhances from anthropology has been unconsidered. This research examined the way how formal structure of productive organizations influences employees' spontaneous cooperation. The paper has the objective to show that the culture inside the organization not only considers formal structure aspect but also other spontaneous group dynamics, different and at the same time complementary to organizational culture management statements. This document is supported on some key concepts such as order, connection and social organization principles which allow presenting a parallel among two types of order: spontaneous order and artificial order. Finally, The way how artificial order restricts, in some cases, social interactions among employees is developed.
\end{abstract}

Keywords: culture, organizational culture, social order, social link, formal group, informal group

El artículo se origina en los resultados de la investigación cualitativa titulada "La estructura formal de organizaciones productivas y su incidencia en la noción de cooperación espontánea”, y desarrollada entre junio de 2006 y septiembre de 2007, en Medellín. Administrador de Empresas (Universidad de Antioquia, 2000), Magíster en Ciencias de la Administración (Universidad EAFIT, 2007). Profesor de la Escuela de Administración y Contaduría Pública, de la Universidad Nacional de Colombia, y miembro del Grupo de Investigación "Emprendimiento y Mipymes". Correo electrónico: dhiguital@unal.edu.co

Estudiante de Administración de empresas, Universidad Nacional de Colombia. Correo electrónico: jclealg@unal.edu.co 


\title{
MICRO CULTURAS E CULTURA ORGANIZACIONAL: CONSTRUINDO A DIALÉTICA NA ORGANIZAÇÃO
}

\section{Resumo}

\begin{abstract}
As disciplinas de administração têm construído um modelo conceitual de cultura organizacional que desconhece o caráter natural que a antropologia cultural outorga ao conceito de cultura. Este estudo procurou investigar como afeta a estrutura formal das organizações produtivas a colaboração espontânea dos trabalhadores. O artigo visa demonstrar que a cultura da organização não só é conformada pelos aspectos da estrutura formal, mas também por outras dinâmicas espontâneas de grupo, complementarias dos postulados sustenidos pela gestão da cultura organizacional. O trabalho se baseia em alguns conceitos-chave como ordem, vinculação e princípios de organização social que permitam estabelecer um paralelo entre duas ordens: espontânea $e$ artificial. Finalmente, é mostrado como a ordem artificial limita, em alguns casos, as interações sociais entre os funcionários.
\end{abstract}

Palavras chave: Cultura, cultura organizacional, ordem social, laços sociais, grupo formal, grupo informa

Higuita, D. \& Leal, J. (2010) Microculturas y cultura organizacional: construcción dialéctica en la organización. En: Revista de la Facultad de Ciencias Económicas de la Universidad Militar Nueva Granada. rev.fac.cienc.econ, XVIII (1)

JEL: M10,M14, M19

\section{Introducción}

Para lograr que los objetivos individuales converjan con los institucionales, en la organización hay reglas, normas y valores establecidos por los directivos que pretenden ser implantados en el comportamiento humano. Los esfuerzos que realiza la dirección para modelar el comportamiento de los empleados se denominan comúnmente "cultura organizacional" y en realidad tan solo constituyen su gestión, ya que como veremos, aquélla se configura por prácticas derivadas de las decisiones del administrador y por la interacción cotidiana entre los trabajadores. Las organizaciones tienen su mayor activo en las personas que las conforman $y$, por lo tanto, se hace necesario entender la manera en que las dinámicas sociales que se dan entre los empleados interactúan con el orden formal que establece la gerencia.

La investigación cualitativa que originó este artículo tenía como propósito fundamental determinar la manera como la estructura formal de organizaciones productivas incide en la noción de cooperación espontánea entre los trabajadores. Es decir, la investigación buscaba comprender las consecuencias de las decisiones y acciones de los directivos sobre la interacción, la cohesión y los vínculos sociales, los cuales se constituyen en factores esenciales para que emerja la cooperación espontánea en la firma (de la cual se hablará posteriormente). En el análisis emergieron dos categorías que comprenden y recogen aspectos a los que hacen alusión los entrevistados: diseño organizacional y discurso dominante de la dirección. Estos aspectos, que intentan dar forma a las actitudes y conductas de los empleados, son algunos de los elementos utilizados para la gestión de la cultura en las compañías.

El artículo tiene por objetivo principal evidenciar que la cultura en la organización no solo se configura por los elementos definidos por los administradores y dirigentes sino que existen en ésta otras dinámicas grupales, distintas a los postulados que maneja la gestión de la cultura organizacional. Adicionalmente, se realiza un análisis de la forma en que la gestión de la cultura impacta dichas dinámicas y la 
manera como éstas complementa la identidad de una empresa particular. Para ello, la discusión se apoya fundamentalmente en dos pares de categorías: orden espontáneo y artificial (Hayek, 1994) y solidaridad orgánica y mecánica (Durkheim, 1967; Giddens, 1998). La comprensión de estas categorías permitirá interpretar mejor dos realidades presentes en la organización: un producto de los postulados de la gestión y la otra, de la interacción entre los sujetos. Igualmente, se abordan autores, como Álvaro Zapata (2007) y Omar Aktouf (2001), quienes han estudiado la manera en que la Administración ha tratado el tema de cultura organizacional, proporcionando así mayores elementos de juicio para el desarrollo del tema.

Por consiguiente, en este artículo se trata la dualidad entre gestión de la cultura organizacional y lo que se ha denominado micro-culturas, y se ha estructurado de la siguiente manera: En primer lugar, se tratan brevemente conceptos como orden social, vínculos, principios de organización social, grupos formales e informales y la noción de cultura desde la antropología. Luego se detalla la metodología empleada en la investigación y la aplicación de técnicas de recolección de información. Posteriormente se describe la manera como la gestión de la cultura organizacional trata de adoctrinar al empleado, y el surgimiento de diversas subculturas en la organización. Finalmente, el texto presenta algunas conclusiones que refuerzan la postura crítica de los autores sobre la forma tradicional de abordar el estudio de la cultura en la empresa.

\section{Sobre el orden social}

Para el economista Friedrich Hayek el orden social, entendido como una multiplicidad de eventos íntimamente relacionados entre sí, puede abordarse desde dos categorías: Taxis y Cosmos. El orden construido y establecido artificialmente por una mente humana que busca uno o varios propósitos definidos y premeditados es denominado Taxis. Mientras que aquel orden social que se configura espontáneamente, y escapa a los pensamientos deliberados del hombre, recibe el nombre de Cosmos. Hayek (1994) se opone a la creencia de que todos los órdenes sociales han sido creados mediante invención deliberada y previa planeación. Las instituciones $u$ órdenes tipo Cosmos son un conjunto de reglas comunes necesarias para la supervivencia social, tales como la lengua, la cultura, la familia, la sociedad, la mone$\mathrm{da}$, etc., que no son el resultado de una intención planeada por una "mente creadora". Por tanto, este tipo de orden emerge espontáneamente y lleva a una convergencia social en las normas que fluye de manera natural; en él nadie planea la forma de ejecutar las acciones para lograr un objetivo definido previamente. En otras palabras, ninguna mente humana decidió como construir tal orden, ya que éste es producto de un proceso indeterminado.

Para Hayek, la organización es considerada como un orden establecido con un fin y unas normas determinadas, en el cual cada una de las partes tiene un lugar y una función asignada para lograr propósitos definidos. Así, el lugar ocupado por cada individuo en una estructura "ha de ser objeto de autoritaria especificación y las normas que a cada cual corresponde obedecer dependen tanto del puesto que ocupen en la organización como de la misión que le haya sido asignada" (Hayek, 1994,91).

Las organizaciones, entonces, son órdenes sociales creados con una finalidad previamente dispuesta y son susceptibles de ser intervenidas mediante diseño, gestión, investigación, planeación, dirección y control. Ahora bien, para lograr la alineación de los objetivos individuales con los objetivos empresariales, la dirección recurre, entre otras prácticas administrativas, a la cultura organizacional, estableciendo unos modos uniformes de comportamiento e interacción para el colectivo de empleados.

\section{Vínculos y principios de organización social}

La interacción entre personas miembros de un colectivo ha sido abordada por distintos teóricos ${ }^{1}$. En particular, la interacción fue objeto de estudio de

\footnotetext{
La interacción social puede entenderse como un proceso en el cual participan al menos dos personas y donde cada una de ellas a través de sus actos genera un efecto y es afectada por la otra; en otras palabras, es una influencia recíproca.
} 
Durkheim quien se propone comprender el funcionamiento de lo social, y plantea que para estudiar la importancia de la diferenciación de la división del trabajo, tenemos que comparar y contrastar los principios según los cuales se organizan las sociedades menos desarrolladas con los que rigen la organización de las sociedades avanzadas (1967). En otras palabras, los principios de una organización social están fundamentados en aspectos diferentes y determinan el tipo de vinculación entre las personas al interior de la sociedad.

De esta manera, Durkheim sostiene que la cohesión social puede establecerse por una solidaridad mecánica o por una solidaridad orgánica, y advierte que en la solidaridad mecánica, los vínculos entre las personas de las sociedades primitivas se configuraban espontáneamente, mientras que en la sociedad industrial, estos vínculos eran determinados racionalmente como consecuencia de la división del trabajo.

Las sociedades en que los principales vínculos de cohesión se basan en la "solidaridad mecánica" tienen una estructura agregada o segmentaria: esto es, se componen de grupos político-familiares yuxtapuestos que son muy semejantes entre sí (...). El conjunto de la tribu forma una sociedad porque es una unidad cultural: porque los miembros de los distintos grupos de clan se adhieren todos al mismo conjunto de ideas y sentimientos comunes (Giddens, 1998,142).

Por consiguiente, en la solidaridad mecánica surge una vinculación producto de la existencia de sentimientos comunes entre las personas. $\mathrm{Y}$ allí existe un conjunto muy firme de sentimientos y creencias compartidos por todos los miembros de la comunidad. Mientras que la solidaridad orgánica establece lo social de manera muy diferente, y no precisamente desde lo común, pues ella no arranca simplemente de la aceptación de un conjunto de creencias y sentimientos comunes, sino de la interdependen- cia funcional en la división del trabajo (Giddens, 1998).

De lo anterior se puede conjeturar que en los espacios colectivos que emergen por medio de la solidaridad mecánica, existen puntos de identificación entre las personas que componen un grupo, permitiéndoles cooperar entre sí $^{2}$; pero esa identificación no implica perder la propia singularidad y el respeto por la misma. En contraste, la solidaridad orgánica, por su carácter artificial, propone una cooperación no a partir de rasgos identificatorios o afinidades entre los sujetos, si no a partir de su utilidad para la organización, generando vínculos mediados por una división técnica del trabajo.

Al igual que Durkheim, Elton Mayo (1977) refiriéndose al paso de una economía social de la aldea hacia el centro industrial moderno, acuña unas categorías semejantes (sociedad adaptable y sociedad establecida) fundamentadas en dos principios de organización, con lógicas distintas entre los miembros del colectivo social, y en las que él percibe un desfase entre habilidades técnicas y habilidades sociales, siendo las primeras más sobrevaloradas que las segundas.

De acuerdo con estos dos principios de organización, la sociedad establecida se cimienta en situaciones de aprendizaje gradual y en las habilidades sociales de las personas. Allí cada persona incorpora progresivamente a su comportamiento los referentes culturales, apropiándose de las creencias, valores y costumbres compartidas que le permiten constituir una identidad cultural. Por su parte, la sociedad adaptable obedece a la necesidad de ajustarse a las exigencias de habilidades técnicas en la industria moderna, para la satisfacción de necesidades materiales particulares.

En las organizaciones contemporáneas es posible observar vinculaciones fundamentadas en la solidaridad tanto mecánica como orgánica, y pareciera

2 En términos generales la identificación puede entenderse como un mecanismo psíquico consciente o inconsciente que induce a un sujeto a comportarse, pensar, vincularse y sentir como otro que actúa como su modelo o encuentra en él afinidad en su comportamiento y el propio. 
que algunos dirigentes solo se interesan por las relaciones racionales que les permitan alcanzar los objetivos económicos, ignorando las relaciones libres y espontáneas que se presentan en los colectivos humanos. Además de las habilidades técnicas presentes en los actos de trabajo, las personas desarrollan relaciones interpersonales que les permiten compartir experiencias favorables o desfavorables, triunfos y fracasos, alegrías y tristezas. Algunas de las prácticas administrativas relacionadas con el talento humano, buscan formar y moldear, a través de principios, reglas, valores, prácticas y mitos, lo que el management tradicional denomina cultura organizacional. De esta manera, los directivos proporcionan estímulos y directrices para programar el comportamiento de las personas, como se verá más adelante.

Desde la posición de algunos gestores de la cultura organizacional, lo necesario para alcanzar los objetivos empresariales es decretar medidas administrativas, desconociendo que los intereses de los empleados podrían estar cimentados en factores diferentes, como lo sugiere Omar Aktouf cuando señala que:

"Si la lógica que guía el funcionamiento de la empresa es económica y racional, la lógica de los miembros de la empresa pertenece a la afectividad y los sentimientos. El trabajo del administrador se ve de esta manera modificado, ya que debe tener en cuenta una organización informal que posee su propia dinámica y sus propios objetivos, que pueden trabar o facilitar el alcance de los objetivos de la organización formal" (Aktouf, 2001,249).

\section{La noción de cultura}

Para algunos teóricos, las ciencias sociales y humanas deben contribuir con la solución de problemas de la sociedad y, por tanto, los resultados y postulados que se han alcanzado con ellas son utilizados de múltiples formas (Chanlat, 2002). En algunos casos, dichos resultados pueden emplearse para mejorar las condiciones de vida, y en otros, para manipular el comportamiento humano en pro de beneficios particulares, haciendo uso indebido de dichas disciplinas y ciencias.

De acuerdo con Ember y Ember (1997), la antropología se ocupa de una gran variedad de preguntas sobre el ser humano, para tratar de explicar características y aspectos de su propia experiencia. En este sentido, la evolución, las formas de pensar y de actuar del hombre como miembro de un colectivo, así como el lenguaje y las tradiciones, son objeto de estudio de la antropología, la cual ve al ser humano como un todo: lo interno a través de la forma de pensar y sentir, y lo externo a través de las prácticas y costumbres de los pueblos.

Ahora bien, no solo se describen los rasgos característicos sobre el modo de vida de un grupo humano sino también las razones de esos rasgos, para comprender por qué una característica es propia de un lugar y no de otros. La antropología, entonces, ayuda al hombre en la comprensión de sí mismo, del otro y del comportamiento humano.

Las posiciones frente a lo que rige el comportamiento del hombre son diversas. Existen posturas que defienden que el comportamiento humano es algo programado y por lo tanto genético. Sin embargo, se sabe que la herencia biológica determina parte del comportamiento, pero no la totalidad de éste y que la noción de comportamiento innato no quiere decir determinismo ni falta de libertad, ya que gracias a la corti-calización del cerebro, el hombre controla sus instintos por medio de su conciencia. Otras corrientes consideran que el comportamiento humano es aprendido y depende del medio social. En este sentido, la cultura es un factor clave en el comportamiento humano, ya que permite definir una identidad grupal. Un ejemplo de ello es que aunque se puede variar una costumbre, en ocasiones se prefiere seguir la norma de grupo, pues ello proporciona seguridad. En el ser humano y de acuerdo con Kottak (1996), los criterios de selección obedecen a patrones de tipo cultural, pero en parte también siguen tendencias innatas.

Pero, ¿qué se entiende por cultura?. De las muchas definiciones que existen de la noción de cultura, se 
dirá que ésta puede entenderse como un conjunto ligado de maneras de pensar, de sentir y de actuar más o menos formalizadas que sirven, de una manera a la vez objetiva y simbólica, para construir a esas personas en una colectividad particular diferenciada. También es la configuración de las conductas aprendidas y el resultado de un comportamiento cuyas componentes y determinantes son compartidos y transmitidos por los miembros de una sociedad particular (Aktouf, 1990).

De acuerdo con Ember \& Ember (1997), la cultura se aprende, es compartida, integrada, adaptativa y cambiante. La cultura es compartida porque si una sola persona hace una cosa, esa cosa es un hábito personal y no un rasgo cultural, pues para que lo sea, ese acto debe ser común a un grupo de personas o a una población. Así mismo, para que algo pueda ser considerado cultural no solo tiene que ser común a la mayoría de la población, sino que tiene que ser algo que se haya aprendido. La cultura es adaptativa y cambiante puesto que una costumbre particular representa la adaptación de una sociedad específica a su entorno. La cultura es mayoritariamente integrada a la comunidad a la que pertenece y por lo tanto no puede pasarse de una sociedad a otra, ya que los elementos y rasgos que la conforman no son una serie de costumbres asociadas al azar, sino que están adaptadas las unas a las otras, o al menos son compatibles.

De los muchos términos usados para describir manifestaciones de cultura, los cuatro siguientes cubren bastante bien el concepto total: símbolos, héroes, rituales y valores (Hofstede, 1999). Ahora bien, el hombre participa simultáneamente de distintos agrupamientos sociales en los cuales comparte reglas y formas de pensar y actuar con otros miembros del colectivo. Uno de esos agrupamientos es la denominada organización contemporánea, la cual se entiende como un colectivo social deliberadamente construido para alcanzar fines específicos.

Los planteamientos anteriores permiten apreciar una relación entre los conceptos sociedad establecida y sociedad adaptable, definidos por Mayo (1977), y los términos solidaridad mecánica y solidaridad orgánica acuñados por Durkheim, y entre tales nociones los denominados grupos informales y grupos formales abordados por la teoría administrativa, que serán tratados a continuación.

\section{Los grupos formales y los grupos informales en la organización}

De acuerdo con la estructura formal (y producto de la división del trabajo), en la empresa se establecen grupos de trabajo con funciones laborales designadas y tareas específicas. En los grupos formales existen medidas para regular el accionar de las personas y orientar el comportamiento hacia los objetivos organizacionales. Existen diferentes tipos de grupos formales tales como grupos de mando, de tarea, equipos inter-funcionales y auto-dirigidos.

En la actualidad es bastante común encontrar en la literatura administrativa el concepto de "equipo" con sus diferentes denominaciones: equipos autónomos, equipos autodirigidos, equipos eficientes. Estos equipos de trabajo son establecidos por la estructura formal con el propósito instrumental de lograr las metas de la manera más eficiente y en donde cada persona dé lo mejor de sí. Por lo tanto, algunas directrices de la organización formal plantean que "si queremos utilizar equipos de forma eficaz debemos comprender por qué funcionan y cómo lo hacen" (Hayes, 2003,12).

La esencia de los grupos formales y los equipos de trabajo es lograr la cooperación entre sus miembros en pro de los fines de la dirección de la empresa. Es claro que allí no interesan las expectativas de las personas ni los aspectos "comunes" existentes entre los empleados, sino únicamente la eficacia.

Adicional a los grupos formalmente definidos por la estructura, en la empresa surgen grupos informales, conformados por individuos con propósitos en común y que se fundamentan en procesos de identificación e interacción. Los grupos informales se constituyen de acuerdo a vinculaciones espontáneas y se presentan en el lugar de trabajo en respuesta a la necesidad de contacto social. En otras palabras, estos grupos se establecen por las afinidades, intereses comunes e identificación entre las personas, 
pues como señala Goffman (1991) solo podemos participar en situaciones sociales si llevamos con nosotros nuestro cuerpo, y este equipo es vulnerable a la acción de los demás.

Es claro que uno de los hallazgos más importantes del trabajo de Elton Mayo (1977), en las primeras décadas del siglo XX, fue los efectos que sobre la producción tenían los grupos informales. Pero eso no es lo fundamental para los trabajadores, pues cuando se trata de realizar tareas existen otros mecanismos, adicionales al contrato y el salario, para que las personas cooperen entre sí, tales como la identificación, la afinidad, la vinculación presente en la denominada solidaridad mecánica y la misma cohesión social.

Las vinculaciones informales que al parecer las personas establecen con las demás en su lugar de trabajo han sido observadas en épocas contemporáneas por Aktouf, quien, refiriéndose al tema de las relaciones humanas en la administración y la contradicción interna existente entre los objetivos de la empresa y los objetivos de las personas, plantea que:

\section{"En la construcción teórica, el grupo informal era identificado como una en- tidad sociológica que funcionaba según la dinámica socio-afectiva y de objetivos propios, paralelos a los de la organiza- ción. En la perspectiva práctica, se vie- ron rápidamente inclinados a descubrir los factores de atracción de las personas para canalizar el funcionamiento del grupo en el sentido de los intereses de los dirigentes empresariales, concebidos como prioritarios y englobadores de los intereses de todas las partes coexisten- tes" (Aktouf, 2001,229).}

Además de lo establecido por la estructura formal, como los dispositivos para la gestión de la cultura organizacional, los reglamentos internos, la escala jerárquica, el puesto de trabajo, etc., en las organizaciones se construye una red de relaciones sociales, personales y espontáneas entre los empleados. Los objetivos y expectativas de las personas, sumados a la interacción social que se da entre ellas, contribuyen con la construcción de esa realidad.

Así entonces, la organización contemporánea cuenta con dos realidades: Por una parte esta la estructura formal que por ser premeditada y construida artificialmente obedece a un orden social tipo taxis. En dicha estructura los vínculos de los trabajadores están definidos por una solidaridad orgánica y obedecen a la lógica de la sociedad adaptable. Por otro lado, está la estructura informal que por configurarse espontáneamente a través de la identificación de rasgos comunes entre los empleados pertenece al orden social tipo cosmos; con características similares a la solidaridad mecánica y a la sociedad establecida.

A continuación se presenta la metodología empleada en la investigación para interpretar el fenómeno cultural en el contexto empresarial, pues como se mencionó anteriormente, este artículo se propuso evidenciar que la cultura en la organización no solo se configura por las acciones y decisiones emanadas en la cima de la jerarquía sino que, adicionalmente, existen otras dinámicas grupales entre los trabajadores con sus propias formas de sentir, pensar y actuar.

\section{Metodología}

Como se precisó anteriormente, el propósito de la investigación fue indagar por los aspectos de la estructura formal de organizaciones productivas que impactan negativamente la cooperación espontánea entre los trabajadores, y para ello, la investigación fue abordada con un enfoque cualitativo, para profundizar y comprender aspectos particulares, cotidianos y subjetivos del contexto empresarial.

Para Galeano (2004), los objetivos, las preguntas y la particularidad del objeto de estudio son fundamentales en la escogencia de las técnicas de recolección y registro de la información. Por consiguiente, el trabajo de campo se organizó de la siguiente manera: en primer lugar, se hizo un acercamiento con el sector industrial para elegir las empresas. Una vez escogidas, se procedió a efectuar el mapeo. Posteriormente, se realizó una fase exploratoria a través 
de observaciones, lo que condujo a desarrollar la etapa final mediante entrevistas. A continuación se describen estas etapas.

La investigación se efectuó en dos organizaciones (una mediana y la otra pequeña) dedicadas a la fabricación y comercialización de productos derivados del plástico, en Colombia ${ }^{3}$. Dichas compañías fueron elegidas por sus condiciones específcas (además de constituir competencia entre sí, son proveedores de almacenes de cadena o grandes superficies) y por su disposición para participar en el estudio.

Al iniciar el estudio, uno de los primeros procesos consistió en realizar un mapeo, es decir, visualizar el escenario en el cual iba a desarrollarse el trabajo de campo, para delinear la aproximación al problema de investigación. En dicho mapeo se identificaron los actores o participantes, los eventos, situaciones, lugares y momentos en los que éstos interactúan, a partir de preguntas de investigación como ¿de qué forma la estructura formal incide en la cooperación espontánea entre los trabajadores?, ¿cómo se configuran los lazos y vínculos entre los empleados al interior de la empresa?, ¿por qué la interacción social es un elemento importante en las relaciones humanas y en la construcción de vínculos sociales?, ¿qué factores definidos o establecidos por los directivos y administradores restringen la interacción social en la compañía? ${ }^{4}$.

De acuerdo con Guber, la única forma de conocer o interpretar en el enfoque cualitativo es participar en situaciones de interacción, donde el investigador debe sumarse a dichas situaciones a condición de no creer que su presencia es totalmente exterior $(2005,48)$. Por consiguiente, para comprender algunos factores que desde lo formal afectan elementos de las dinámicas grupales como la cooperación espontánea, se necesita algún tipo de interacción entre el investigador y los participantes del entorno organizacional. Para dicho fin se empleó la observación como técnica de recolección de información ya que ésta implica focalizar la atención en aspectos de la realidad bajo estudio, tratando de captar sus elementos constitutivos y la manera como interactúan entre sí. De esta manera, se iniciaron las primeras visitas de observación a las empresas, para abordar y explorar progresivamente el contexto social y efectuar, seguidamente, una profundización en el mismo.

En el trabajo de campo se realizaron seis visitas de observación, de cuatro horas cada una, en distintos días de la semana y a diferentes horas del día para captar variados momentos de la vida organizacional, dado que, como afirma Ander-Egg (1994), con dicha técnica la intención es estudiar fenómenos sociales naturales no provocados artificialmente, como el caso de la cooperación espontánea y de otras dinámicas grupales derivadas de la interacción social.

¿Por qué se empleó la observación? Porque 1) permite tener un mayor acercamiento al objeto de estudio cuando la intención es comprenderlo, como en este caso; 2) porque esta técnica ayudaría a identificar informantes claves para ser entrevistados; y 3) porque las metodologías cualitativas conducen a estudiar eventos en su contexto natural, intentando dar sentido a los fenómenos de acuerdo con los significados que tienen para las personas implicadas. Si bien no se efectuó una observación participante total, en el sentido de asumir los comportamientos de los trabajadores, si se desarrolló una observación participante en cierto grado, porque la naturaleza del problema (efectos de elementos derivados en la estructura formal sobre dinámicas grupales y vínculos sociales) así lo ameritaba. Dicha observación fue participante porque el investigador interactuaba con los empleados compartiendo con ellos periodos y eventos laborales ${ }^{5}$, dado que el uso del lenguaje

3 De acuerdo con el compromiso pactado con las empresas, sus nombres se mantienen en reserva.

4 Es importante precisar que el enfoque cualitativo de investigación es abierto. En este caso particular, se inició con algunas preguntas preliminares, pero durante el mismo proceso fueron apareciendo otros interrogantes. Lo mismo sucede con las categorías de estudio.

5 Los procesos de observación permitieron indagar por políticas institucionales, normas, puestos de trabajo, reglamentos, relaciones de autoridad y de comunicación (aspectos formales). Igualmente se preguntó por la cooperación, relaciones directas entre compañeros, diálogo y expectativas de los empleados en su trabajo (elementos informales). 
como herramienta de investigación social resalta la importancia de conocer los signos que una persona recibe de otros miembros del discurso común y el cual contribuye a su competencia como un oyente de conversaciones diarias (Cicourel, 1982).

Es pertinente recordar que en un proceso de investigación cualitativa, el investigador transforma e incide en la conducta de los informantes cuanto mayor sea su interacción con ellos. En las primeras observaciones, la presencia del investigador representaba un elemento extraño en el espacio laboral. Sin embargo, el observador fue ganando familiaridad, lo que facilitaba que los empleados se presentaran con mayor naturalidad.

La utilización de notas de campo como técnica de registro de datos fue especialmente útil porque permitieron abordar gradualmente las categorías preliminares del estudio. Durante la misma jornada de observación, el investigador destinaba un momento para asentar, en las correspondientes fichas de observación, la información obtenida en la planta de producción, en la bodega, en las oficinas y en la cafetería de la organización. Este registro se realizó a mano, tratando de que lo anotado fuera lo más "nativo" posible, es decir, escribiendo los términos tal cual habían sido escuchados, evitando una inadecuada interpretación por parte del investigador. En esta fase exploratoria, la revisión de la literatura se empleó para depurar conceptualmente las categorías que fueron emergiendo con la información generada durante el proceso ${ }^{6}$. Para lograr validez, Aktouf (2001) recomienda verificar con preguntas o entrevistas los elementos observados así como clasificar las versiones de diferentes informantes sobre un mismo evento.

Para profundizar en el propósito de la investigación, se vio la necesidad de acompañar la observación con entrevistas semiestructuradas ${ }^{7}$, puesto que la entrevista es una técnica eficaz para obtener datos relevantes y significativos desde el punto de vista de las ciencias sociales, para averiguar hechos, fenómenos o situaciones sociales (Ander-Egg, 1994). La siguiente fase se concentró, entonces, en elegir los participantes para validar y contrastar la información proveniente de las observaciones. Ahora bien, considerando que las razones de selección de los informantes son de comprensión y de pertinencia y no de representatividad estadística, en la elección de los trabajadores se incluyeron cuatro criterios de importancia cualitativa: el cargo dentro de la jerarquía (parte administrativa y parte operativa); su perspectiva hacia la organización (trabajadores de diferentes áreas, de poca y mucha antigüedad, hombres y mujeres); la relación con el problema de investigación (es decir, empleados clave por la información que poseían); y su disposición para participar en el estudio. Inicialmente se entrevistó un grupo preliminar de nueve personas, de acuerdo a la conveniencia y disponibilidad manifestadas durante las observaciones. Es decir, fue la etapa de observación (realizada anteriormente), la que facilitó identificar actores sociales clave en cada organización. Posteriormente, se fue configurando un nuevo grupo conformado por ocho personas más, de acuerdo a la información suministrada por los empleados del primer grupo, lo que permitió profundizar y validar las categorías emergentes.

La etapa final consistió en construir las categorías de análisis para interpretar los resultados a la luz de los planteamientos del marco teórico. La interpretación es una característica fundamental de los estudios cualitativos, donde el investigador integra y reconstruye diversos aspectos los cuales no tendrían sentido si fueran tomados en forma aislada como constataciones empíricas. De este modo, en un primer momento se le dio un nombre a fragmen-

6 Tanto la información recolectada en fichas como la proveniente de entrevistas fue registrada, sistematizada y almacenada respectivamente.

7 Aquella entrevista en la cual el investigador no se ciñe a un listado de preguntas rígido ni sigue un orden estricto y estandarizado al momento de hacer las preguntas. Algunos de los interrogantes de las entrevistas apuntaban a indagar por la actitud (y correspondiente acción) de los empleados cuando sus compañeros de la misma sección y de otras secciones, tenían algún problema o una necesidad. También se preguntó por las prácticas, costumbres y valores grupales, por las relaciones laborales con compañeros, por el significado de las decisiones administrativas y de las interacciones sociales para los trabajadores, entre otros aspectos. 
tos de información que empezaban a aparecer con frecuencia en las entrevistas, y que son elementos que afectan la interacción, los vínculos sociales y por tanto, algunas dinámicas grupales de la organización. De este análisis e interpretación emergieron dos categorías: diseño organizacional y discurso dominante de la dirección. La primera categoría comprendió puesto de trabajo, jerarquía, normas y departamentalización. La segunda está conformada por administración del tiempo, productividad y separación de las esferas laboral y privada. Estas categorías, aspectos formales en la organización, son dos maneras particulares de gestionar la cultura organizacional, que tienen impacto en la interacción ${ }^{8}$.

\section{Discusión}

Para lograr que los empleados se alineen con los propósitos de la empresa, algunos administradores proporcionan directrices, acciones, decisiones mecanismos, que permiten gestionar la llamada cultura organizacional. Se trata de hacer al empleado a imagen y semejanza de la compañía, pues el propósito es cohesionar la fuerza de trabajo y adherir el personal con los valores, los principios y la estructura formal. Para la corriente funcionalista de la administración uno de los caminos para cambiar a las organizaciones es modificar su "cultura", es decir, cambiar los sistemas dentro de los cuales viven y trabajan las personas. Dicha corriente considera que una cultura dominante expresa los valores centrales que comparten los miembros de la empresa. Desde esa perspectiva, se asume que son los significados compartidos de la cultura lo que la hace un mecanismo tan poderoso para encauzar la conducta. Para la dirección funcionalista, la gestión de la cultura organizacional es necesaria porque contribuye a amasar y moldear el comportamiento de los trabajadores $y$ porque ofrece a directivos la forma de influir en los demás, ya que establece normas que deben ser acatadas por los empleados y que sirven para controlar a las personas. "Se considera que el control ejercido por las directrices, los consejos, las reglas y las leyes es más notorio que el control ejercido por las contingencias mismas, en parte porque es menos sutil. Y, por lo tanto, parecería que este último representara una mayor contribución personal y un mérito interno" (Skinner, 1977,118).

Para la administración tradicional, lograr los objetivos de la empresa es una manera de realizar los objetivos personales y, en este sentido, la cultura facilita la aceptación de un compromiso con algo que supera las expectativas personales. "La cultura es el aglutinante social que mantiene unida la organización al darle los criterios apropiados sobre lo que los empleados deben decir y hacer. Esta sirve como un mecanismo que crea sentido y permite el control, que orienta y da forma a las actitudes y comportamientos de los empleados" (Robbins, 2004,528). Por lo tanto, las directrices están dirigidas a reforzar o limitar el comportamiento, en procura de edificar la cultura organizacional para que los trabajadores se sientan más comprometidos y se integren a la organización.

De manera irreflexiva los teóricos de la administración y de la organización han formulado brusca $e$ inadecuadamente la existencia de una supuesta cultura de la empresa que compartiría la naturaleza de los órdenes sociales del tipo taxis, es decir, de las agrupaciones humanas susceptibles de ser diseñadas a voluntad (...) y controlables en su funcionamiento (López, 2004,20).

Algunas medidas administrativas buscan formar la cultura a través de principios, reglas, valores y prácticas, muchas de las cuales quedan expresadas en los manuales, los reglamentos, misión, visión y valores corporativos. De acuerdo con la teoría administrativa, una cultura fuerte tendrá una gran incidencia en el comportamiento de sus miembros, porque los empleados internalizan las normas y guías. No obstante, la administración tradicional parece no tener en cuenta que para que algo pueda ser considerado

8 Considerando que los propósitos del presente artículo se centran en abordar elementos de la gestión de la cultura y de las microculturas en la organización, los hallazgos sobre discurso dominante de la dirección y parámetros de diseño organizacional se tratan muy brevemente. 
cultural no solo tiene que ser común a la mayoría de la población, sino que tiene que ser algo que hayan aprendido.

A través de lo que dicen y hacen, los altos ejecutivos establecen normas que se filtran a toda la organización, sobre cómo hay que vestirse, que actividades son premiadas con aumentos, ascensos y otras recompensas. Si el interés de la administración es la productividad y la rentabilidad, pondrá todo lo que tenga a su alcance para lograrlas, tomando planteamientos que desde otras disciplinas se han elaborado y aplicándolos al mercadeo, las finanzas y la gestión humana. Y es lo que la administración ha hecho con la cultura, haciendo un uso instrumental del concepto, pues la pobreza de los desarrollo teóricos de carácter explicativo ha favorecido la emergencia de teoría prescriptiva no fundamentada epistemológicamente, que normatiza sobre la forma en que deber ser gerenciada la cultura para apoyar el alcance de los propósitos organizacionales (López, 2004).

Los gestores de la cultura organizacional establecen postulados sin fundamentos epistemológicos cuando tratan de introducir prácticas y valores corporativos en los empleados, pues la conducta de una persona está solo parcialmente determinada por las normas de su entorno, ya que el individuo posee la capacidad para desviarse de ellas y reaccionar de maneras nuevas, creativas o inesperadas.

Como se mencionó anteriormente, el discurso dominante de la dirección y los parámetros del diseño organizacional son dos herramientas utilizadas para gestionar la cultura, que emergieron durante la investigación adelantada. Con la administración del tiempo, los empleados sienten que no pueden dedicar algunos minutos en colaborar con sus colegas dado que corren el riesgo de no cumplir con sus estándares, inclusive en labores que no requieren altísima concentración, viéndose en peligro su evaluación y remuneración. La exigencia de productividad y competencia lleva a que puedan romperse los vínculos entre las personas, generándose rivalidad entre éstas, porque algunos empleados harán lo que esté a su alcance para competir con sus compañeros. Con el discurso que busca separar tajantemente la vida personal de la laboral se limita la interacción y la posibilidad de construir vínculos sociales al interior del pequeño grupo de trabajo. Se desconoce que es mucho más fácil pedir ayuda en un problema laboral a una persona cercana que a alguien que sólo se lo conoce de nombre en un organigrama.

Efectos similares sobre la interacción, los vínculos, la cooperación espontánea, las prácticas, valores y relaciones al interior del grupo (que cuenta con sus propias formas de sentir, actuar y compartir) se derivan del diseño organizacional. Sin embargo, no se busca desacreditar los logros que permite la división técnica del trabajo y su correspondiente especialización. No se trata de eliminar las jerarquías cuando traen eficacia. No se intenta crear organizaciones sin normas ni reglamentos o empresas donde no exista una clara definición y diseño del puesto de trabajo. Se trata, en primera instancia, de reconocer los impactos que estas medidas del diseño organizacional tienen en otras esferas y ámbitos organizacionales, y en segundo lugar, de propiciar espacios de reflexión para los diseñadores, ingenieros y directivos.

De otro lado, para lograr la eficacia y la eficiencia se pretende que los trabajadores se identifiquen con los objetivos organizacionales mediante la alineación. La alineación organizativa es un concepto que se refiere a la extensión por la cual la estrategia y la cultura de la organización se combinan para alcanzar los objetivos dispuestos por la estrategia. Es decir, mediante la alineación se crean ensamblajes entre las recompensas, los sistemas de resultados y las prácticas administrativas, que redunden en beneficio de la empresa, pues como sostienen Aubert \& Gaulejac (1993) se trata de hacer querer más que de hacer hacer, no se trata de humanizar las normas sino de normalizar al hombre. En otras palabras, a la gestión de la cultura organizacional parece que solo le interesa aquel empleado que hace "suyos" los intereses y fines organizacionales. Prueba de ello es que el proceso de selección, la evaluación del desempeño, las actividades de capacitación y los procedimientos de ascensos buscan garantizar que los contratados se adapten a la cultura, premian a quienes la respaldan y castigan a quienes la ponen en tela de juicio. 
Sin embargo, cuando los antropólogos consideran algo como cultural, siempre existe la variación individual, lo cual significa que no todos los miembros de una sociedad comparten un rasgo cultural concreto (Ember \& Ember, 1997). Pero al parecer, algunos administradores no comprenden esto y pretenden homogeneizar y normalizar a los empleados, mediante reglamentos, capacitaciones y normas que apuntan a sistematizar el comportamiento humano, para que todos los empleados sonrían y saluden de la misma manera. La productividad y la rentabilidad como objetivos macro de la empresa pueden convertir en medios, a los demás elementos del sistema. Incluso a las personas, para las cuales se diseñan modelos, moldes, sobre el comportamiento anhelado por la dirección.

La administración funcionalista intenta partir de prácticas como la socialización para moldear las formas de sentir y de pensar de los empleados. Cuando las expectativas del empleado y la realidad difieren, el nuevo empleado debe pasar por una socialización en la que se desprenda de sus concepciones antiguas y las sustituya con otras que la organización juzgue más deseables. No obstante, quienes han estudiado seriamente la cultura consideran que ésta "supone captar su carácter normal sin reducir su particularidad. Las descripciones de la cultura deben realizarse atendiendo a los valores que los diferentes pueblos asignan a las cosas, atendiendo a las fórmulas que ellos usan para definir lo que les sucede" (Geertz, 2005).

Desde nuestra posición, la gestión de la denominada cultura organizacional propicia condiciones para la sumisión de los miembros de la organización mediante conmovedores discursos, pancartas, cursos y rituales que definen esa particular forma de ser, de sentir y de pensar colectivas. La cultura organizacional, como la piensan y diseñan algunos directivos, ejerce cierto grado de influencia sobre el comportamiento del individuo y el grupo, pretendiendo anular su libertad, desconociendo la individualidad y la pluralidad propias del ser humano $e$ ignorando que comportamiento innato no quiere decir necesariamente determinación y, por tanto, falta de libertad. En otras palabras, el hombre tiene capacidad de control y, en ocasiones, de decisión al interior del grupo social al que pertenece. Recuérdese que el comportamiento humano se deriva de elementos programados y elementos culturales, en una combinación única y dinámica, pues la cultura se presenta en distintos aspectos interrelacionados como un hecho social total, donde se observa:

Interdependencia entre historias y estructuras; dialéctica entre vida económica, social y simbólica; conjunto englobante de las diversidades o de las separaciones; representaciones colectivas que ligan materialidad e inmaterialidad; finalmente, conjunto construido alrededor de elementos básicos universales como el mito (Aktouf, 1990,19).

Sin embargo, no podría afirmarse que no existe cultura al interior de una empresa particular. La organización como colectivo humano, conformada por grupos de personas que están en permanente contacto unas con otras, compartiendo metas y formas de trabajar, construyendo su realidad específica, genera ciertos hábitos, valores y costumbres propias del grupo o de la organización, y que no necesariamente corresponden al ideal del administrador, porque entre colectivos humanos existen reglas que podrían denominarse inconscientes.

La interacción diaria de los trabajadores en la empresa lleva a la construcción de "microculturas", con sus propias premisas, supuestos y prejuicios no declarados, como reflejo de la dimensión social de las personas. En la empresa existen múltiples grupos, con sus reglas internas, con afinidades en los modos de pensar, actuar y trabajar que se traducen en pequeños ambientes de trabajo específicos, y ello deriva en la aparición de microculturas, que en algunas ocasiones se dan por áreas o unidades. De esta manera, al comparar lo que sucede en la fábriLa interacción diaria de los trabajadores en la empresa lleva a la construcción de "microculturas", con sus propias premisas, supuestos y prejuicios no declarados, como reflejo de la dimensión social de las personas. En la empresa existen múltiples grupos, con sus reglas internas, con afinidades en los modos de pensar, actuar y trabajar que se traducen en pequeños ambientes de trabajo específicos, y ello deriva en la aparición de microculturas, que en algunas ocasiones se dan por áreas o unidades. De 
esta manera, al comparar lo que sucede en la fábrica con lo que puede suceder en el área de finanzas de la misma organización, Etkin (2000) sostiene que como grupos, ellos crean sus pautas de relación sobre diferentes bases, por ejemplo el valor de ser solidarios o el ser competitivos. Estas microculturas se dan por la conformación de vínculos semejantes a los existentes en el tipo de solidaridad mecánica, de la cual se habló anteriormente. La cultura de una compañía, entendida como su identidad, se constituye tanto por las decisiones y acciones de los directivos como por esa dinámica específica y espontánea que surge entre los miembros del grupo. Por ejemplo, aunque se observó que la estructura formal establecía premios para las personas más productivas, en el grupo preferían a los compañeros más leales, con los cuales surgía espontánea y recíprocamente una especial cooperación producto de su particular vinculación.

En la práctica son pocos los dirigentes que buscan gestionar la cultura organizacional teniendo en cuenta las necesidades e ideas potenciales que pueden obtener de su grupo de trabajo. La organización formal (orientada por los dirigentes) intenta sobreponerse a la informal desconociendo y excluyendo algunos elementos fundamentales para los seres humanos e importándole solamente la productividad.

Para la ideología dominante en los medios administrativos, todo problema debe ser considerado siempre a partir de la prioridad determinada por la racionalidad económica (...) El énfasis puesto sobre la rapidez de ejecución en detrimento de otros elementos de desempeño y del rendimiento, tiene por efecto reducir los intercambios a su expresión más elemental y más frustrante (Chanlat \& Bédard, 1990,15).

Es decir, la administración funcionalista considera que la organización informal trae desventajas para los intereses de los accionistas porque se desarrollan rumores indeseables, debilita la motivación y satisfacción, fomenta la resistencia al cambio y opera fuera del control de los administradores. Para Davis \& Newstrom (2003), multiplicadores del paradigma funcionalista, lo que es bueno para los empleados no siempre lo es para la organización. Aunque las pautas para café son aconsejables, si el empleado dedica 15 minutos más de lo debido a socializar por la mañana o por la tarde disminuirá la productividad, lo cual redunda en perjuicio de la compañía.

Con tal planteamiento se está confinando la posibilidad de diálogo, porque se cree que ello no contribuye con la productividad de la compañía, ya que para muchos directivos, los espacios y momentos que los trabajadores dedican a realizar intercambios verbales son considerados como una pérdida de tiempo y de dinero para la empresa. La gestión tayloriana del trabajo, preocupada por la haraganería, se dedicó a transformar al obrero en un autómata sin pensamiento, ejecutante estricto de tareas fragmentarias, dirigido por el cronómetro, el salario, las políticas.

Sin embargo, Chanlat \& Bédard señalan que los impedimentos al diálogo y la socialización son naturalmente superados en los grupos informales: En sus tertulias, los miembros del grupo se dan mutuamente ejemplos de los comportamientos a seguir o a proscribir. Las personas se construyen y se refuerzan mutuamente en sus creencias individuales y colectivas. En este escenario, esas actividades [de vinculación] juegan un papel fundamental para mantener y desarrollar la identidad de cada uno (1990).

Es evidente que, cuando sólo importa la eficiencia, los aspectos más sobresalientes de la organización informal podrían ser objeto de manipulación por los directivos, buscando que no perturben los objetivos organizacionales. De esta forma lo incita Herbert A. Simon en su texto El comportamiento administrativo (1972) cuando afirma que la estructura formal no realiza ninguna función, a menos que limite realmente las relaciones no formales que pueden desarrollarse dentro de ella. Pareciera que Simon olvida que como ser complejo, el hombre construye relaciones complejas con los demás a través de la interacción comunicativa y que, por lo tanto, la vida en la organización es más que una simple estructura jerárquica con unos propósitos escritos. Por ejemplo, los intercambios de información en las organizaciones estudiadas obedecían a dos dinámicas. En unos casos, estaban mediados por relaciones formales (como cartas, pancartas, órdenes, etc.), y en los otros, eran abiertos y espontáneos producto 
de las actividades extralaborales o laborales de los trabajadores.

Como se mencionó anteriormente, se podría hacer una analogía entre organización informal y lo que Hayek llama orden espontáneo, en el cual su existencia está basada en relaciones puramente abstractas que no son producto de creación intencionada. La organización informal no está supeditada o limitada a la mente del director o a los postulados de la gestión de la cultura organizacional, y en consecuencia, cuando algunos directivos buscan manipular la informalidad y las relaciones e interacciones libres y espontáneas entre los trabajadores, atentan contra sus propias características e incluso pueden llegar a impactar los vínculos que los empleados han construido entre sí. Prueba de ello son los efectos que tienen el discurso de la dirección y los parámetros de diseño organizacional sobre la interacción y la cohesión social.

Una cultura, como resultado de la interacción de los individuos en la sociedad, es una entidad que comparte las características de los órdenes sociales no planeados ni diseñados previamente para objetivos específicos. Precisamente, las microculturas surgen en la empresa de forma natural, producto de la interacción de los trabajadores y sus propias dinámicas sociales. Una muestra de ello es que en cierta ocasión, por iniciativa propia, un empleado se dirigió hasta una compañera para ayudarla en su trabajo, cuando pudo haber esperado a que el supervisor fuese quien la ayudara. En ese momento no era la cultura corporativa establecida en la organización la que gobernaba la acción del trabajador, sino que ésta era dirigida desde su interior, por el contacto social entre compañeros de trabajo, y por los vínculos establecidos entre ellos en las actividades realizadas a través del tiempo. Al respecto Goffman argumenta que:

Los individuos, en presencia de otros, se encuentran en una posición ideal para compartir un mismo foco de atención. Esto es, en combinación con su capacidad para indicar sus cursos de acción física y ajustar sus reacciones a indicaciones similares de los demás (...), sea como apoyo de tareas altamente colaborativas o como forma de acomodar tareas adyacentes. El habla aumenta inmensamente la eficacia de tal coordinación (Goffman, 1991,176).

Es decir, que la ayuda directa entre los trabajadores que comparten una microcultura, da como resultado una convergencia de la fuerza individual con una alianza grupal, lo que permite potencializar el desempeño productivo y probablemente la relación informal que han entablado los individuos a partir de su simpatía social. El comportamiento humano es fruto de la interacción de los individuos en un espacio y en un momento dado, como puede apreciarse en el siguiente fragmento:

Al terminar el turno de trabajo, los empleados se dirigían hacia la cafetería, pues aquel lugar representaba un espacio de encuentro con el otro. Un espacio en el cual las personas interactúan espontáneamente y se apoyan de manera abierta y sincera. Para ellos es un proceso que hacen inconscientemente, pero lo hacen. Mientras se organizaban, se observaba entre ellos un diálogo más abierto a través del cual se invitaban a compartir algunos momentos después del trabajo9. Aunque aún estaban bajo el techo de la organización, eran momentos y espacios donde los empleados empiezan a mostrarse tal como son, reflejando la existencia de vínculos particulares entre ellos, distintos a los observados en la planta de producción. Muy probablemente en aquel lugar no exista ninguna directriz formal que defina su comportamiento. El grupo social es generador de sus propias pautas de acción y de su propia microcultura, donde los intereses particulares se articulan con los intereses comunes, hay analogía de creencias y cada persona se adhiere por convicción a las dinámicas de interacción en su respectivo grupo, ya sea de forma verbal o conductual. Aquí, las personas buscan fortalecer sus lazos llevando a cabo actividades voluntarias que les proporcionan satisfacción.

9 Con el término organizaban, el autor quiere significar todo el proceso que realizan los empleados de una planta de producción al finalizar su jornada de trabajo y prepararse para salir de la empresa. 
Aun cuando en la organización se establece una cultura (que a través de la imposición de comportamientos restringe en cierta forma la relación entre los sujetos), como proceso natural, los trabajadores encuentran más afinidades con algunos compañeros que con otros. En lugar de entorpecer el trabajo, las microculturas pueden mejorar y facilitar la productividad gracias a la rapidez en la información y a la camaradería, las cuales contribuyen para que los empleados se ayuden unos a otros. En las entrevistas, algunos trabajadores manifestaban que ante una dificultad de un compañero, con el cual tienen una vinculación y/o afinidad especial, por supuesto que le colaborarán porque sienten el deseo de hacerlo, porque es algo que les nace y sienten la satisfacción de realizarlo, de ayudarse entre sí. De igual forma, otro empleado manifestó que: por los años que llevamos trabajando juntos y por la relación que tenemos, con Esteban nos entendemos mucho para trabajar, a veces incluso nos entendemos hasta los gestos y yo veo que hacemos las cosas más fáciles ${ }^{10}$.

Cuando los trabajadores logran entablar y fortalecer una relación fluida de respeto, colaboración, receptividad, comodidad y armonía personal, dicha relación genera un impacto en el ámbito laboral ya que al sentir que pueden ser apoyados por las personas que los rodean, los trabajadores estarán más abiertos para afrontar su compromiso de ayuda con los compañeros y por ende con la organización. De esta forma lo deja entrever Zapata cuando sostiene que "la cultura organizacional es un esquema de referencia para patrones de información, comportamiento y actitudes que compartimos con otros en el trabajo y que determinan el grado de adaptación laboral; en esta medida representan un aprendizaje continuo en el cual la cultura organizacional se enriquece con los aportes de los individuos, se perpetúa a través de ellos a la vez que las personas enriquecen sus otros entornos" (Zapata, 2007,173).

Cuando en pro de la rentabilidad y la productividad los dirigentes gestionan la cultura organizacional y sus respectivas normas de comportamiento, se puede ver afectada la interacción entre los empleados, su vinculación y la posibilidad de que surja la cooperación espontánea entre los trabajadores. La organización informal, los grupos informales y las vinculaciones e interacciones abiertas entre los trabajadores, no sólo son aspectos que no estarían siendo contemplados por la gestión de la cultura organizacional, sino que son elementos de vital importancia para el estado anímico de los empleados, para el buen clima laboral y para la consecución de los objetivos organizacionales.

Pareciera que, mientras pueda, la mirada funcionalista de la administración intentará anular o limitar aquellos aspectos informales entre las personas. No obstante, de acuerdo con las ciencias humanas fundamentales, se ha señalado que la subjetividad y los símbolos que interpreta todo ser humano, poco se pueden "manipular" exteriormente, pues depende de "la coherencia entre la vida material, concreta, y la vida simbólica representacional, como elementos interdependientes de un todo. La cultura no puede ser considerada como una ciencia experimental en busca de leyes, sino una ciencia interpretativa en busca de significaciones" (García, 2007, p. 165). Por tanto, podemos decir que la cultura es una configuración dinámica, espontánea, integradora de los valores, prácticas y percepciones de un colectivo, en la cual no se anula ni minimiza la individualidad de cada persona.

\section{Conclusiones}

Algunos directivos ignoran que los trabajadores no actúan ni reaccionan aisladamente como individuos, sino como miembros de un grupo, que tienen un comportamiento social y valoran la interacción, la posibilidad de dialogar y los vínculos que han construido con sus compañeros.

Las microculturas se presentan porque los trabajadores tienen propósitos, intereses y afectos comunes o incluso afinidades en los modos de pensar, trabajar o vivir, lo cual las lleva a agruparse, a compartir espacios y a construir y afianzar vínculos

10 Entrevistas de la investigación. 
entre ellas. Y ¿por qué se genera esta comunión de intereses y afectos? Porque la interacción y la cohesión social posibilitan una cierta identificación, la cual contribuye a propiciar la simpatía entre los empleados. La identificación puede surgir siempre que el sujeto descubre en sí un rasgo común con otra persona. Dicha identificación lleva en muchos casos a la realización de actividades comunes en pro de un beneficio compartido, sin que cada persona pierda su propia identidad.

No se puede desconocer que estos pequeños grupos definen sus propias reglas de comportamiento, sus recompensas o sanciones sociales, sus objetivos, su escala de valores, creencias y expectativas, y que, en la mayoría de los casos, reflejan una realidad social distinta a la planeada por la gestión cultural. Esos colectivos humanos tienen su cultura porque comparten prácticas, valores, modos de pensar y sentir, sentimientos e ideas, de una manera específica.

Las prácticas que se enmarcan en la gestión de la cultura organizacional no están considerando la individualidad y la pluralidad de los trabajadores. Solo importa que los empleados interioricen los valores y los mitos de la empresa, para que todos estén uniformados, exterior e interiormente, y caminen por donde manda la dirección. Para ello, los gestores de la cultura utilizan los símbolos, el lenguaje, el discurso, el diseño y otras medidas administrativas. La mirada utilitarista de la administración ignora, tal vez deliberadamente, que la cultura es algo dinámico y que se construye con la diversidad. Olvida que nace en una colectividad en la cual unos miembros reconocen a los otros como iguales. Ninguna cultura es posible sin identificación con pares y lugares de socialización reconocidos como tales, sin interiorización de esta situación como fundadora de lo que el miembro de la colectividad es, o cree que es (Aktouf, 1990).

Los directivos y gestores de la cultura en la empresa quisieran moldear la personalidad y pretenden adoctrinar a los empleados, para hacerlos creer en lo que la administración quiere que crean, olvidando que la verdadera cultura organizacional se construye por la dialéctica entre los miembros de ese colectivo humano denominado organización.
En la organización informal emerge una dinámica grupal con sus propios rasgos culturales que contribuyen a definir la identidad de una empresa particular. Esta dinámica favorece el ambiente de trabajo, las relaciones humanas, la comunicación directa y las dificultades laborales. Esta realidad social se presenta producto de la interacción (abierta y cotidiana) que favorece la aparición de vínculos sociales entre los sujetos, los cuales a su vez propician la cooperación espontánea en la empresa. Y dicha realidad social puede ser comprendida en su esencia mediante el uso de metodologías cualitativas, donde los investigadores se sumergen en los contextos empresariales $\mathrm{y}$, a través de la interacción directa con los agentes o participantes, se puede interpretar o comprender el fenómeno organizacional.

Algunos empleados desean cooperar entre ellos por el vínculo que han construido a lo largo del tiempo, o por la posibilidad de compartir habilidades, destrezas, formas de dialogar y de dar solución a las dificultades laborales. Pero ello suele ocurrir cuando no se restringe totalmente la posibilidad de dicho intercambio entre los trabajadores, cuando no se coarta la interacción social, y se permite, por el contrario, la creación de espacios comunicacionales y de integración de los empleados.

Los administradores requieren, entonces, dar espacio a las manifestaciones naturales que surgen en las personas, y no tratar de restringir las características de las microculturas porque estas complementan las formas particulares de pensar, actuar y modos de trabajar de los empleados. Es importante precisar que no se busca sobredimensionar lo informal y subestimar lo formal, pues en la organización se requieren tomar medidas administrativas que propendan por la productividad.

Es claro que si la empresa no mantiene o mejora su productividad, pone en riesgo su supervivencia económica y algunos empleados terminarán perdiendo su empleo. Lo ideal es que con la productividad no sólo gane la empresa sino también los trabajadores, y que en el interés de diseñar la cultura organizacional no se restrinja la dinámica grupal donde los empleados satisfacen parte de sus necesidades sociales como la solidaridad, la interacción y el diálogo, 
y comparten valores, símbolos y hasta ritos. Para una buena gestión se necesita también interpretar y comprender la vida organizacional, y si bien algunas relaciones informales pueden, en ciertas ocasiones, obstaculizar los propósitos organizacionales, también favorecen los objetivos de la organización a través de la cooperación espontánea que en general emerge entre las personas que comparten valores en común, al interior de un colectivo.

\section{Referencias}

Aktouf, O. (1990). El simbolismo y la cultura organizacional. De los abusos conceptuales a las lecciones de campo. Québec: Pul-Eska. Traducción al español.

Aktouf, O. (2001). La administración. Entre la tradición y la renovación. Cali: Universidad del Valle.

Ander-Egg, E. (1994). Técnicas de investigación social. México: El Ateneo.

Aubert, N. y Gaulejac, V. (1993). El coste de la excelencia. Barcelona: Paidós.

Chanlat, A. y Bédard, R. (1990). L'individue dans L'organisation, les dimensions oubliés. Québec: Pul-Eska. Traducción libre de Rodrigo Muñoz.

Chanlat, J. F. (2002). Ciencias sociales y administración. Medellín: Fondo editorial Universidad Eafit.

Cicourel, A. (1982). El método y la medida en sociología, Madrid: Editora Nacional. pp. 14, 18, 26, 243 y 236

Davis, K. y otros. (2003) Comportamiento humano en el trabajo. México: McGraw-Hill.

Durkheim, E. (1967) La división del trabajo social. Buenos aires: Schapire.

Ember, C. y Ember, M. (1997) El concepto de cultura. En: Antropología cultural. Madrid: Prentice Hall.

Etkin, J. (2000) Política, gobierno y gerencia de las organizaciones: acuerdos, dualidades y divergencias. Buenos Aires: Prentice Hall.
Galeano, M. E. (2004) Diseño de proyectos en la investigación cualitativa. Medellín: Universidad Eafit.

Garcia, O. H. (2007). "La cultura humana y su interpretación desde la perspectiva de la cultura organizacional". En: Pensamiento \& gestión, Universidad del Norte, volumen (22): 143-167.

Geertz, C. (2005). La interpretación de las culturas. España: Gedisa.

Giddens, A. (1998). El capitalismo y la moderna teoría social: un análisis de los escritos de Marx, Durkheim y Weber. España: Idea books.

Goffman, E. (1991). La presentación de la persona en la vida cotidiana. Buenos aires: Amorrortu.

Guber, R. (2005) La etnografía. Bogotá: Norma.

Hayek, F. (1994). Derecho, legislación y libertad, unión editorial. Madrid: Unión Editorial.

Hayes, N. (2003). Dirección de equipos de trabajo, una estrategia para el éxito. Madrid: Thomson.

Hofstede, G. (1999). Culturas y organizaciones. El software mental, la cooperación internacional y su importancia para la supervivencia. Madrid: Alianza Editorial.

Kottak, P. (1996). Antropología, una exploración de la diversidad humana. Santa Fé de Bogotá: McGraw Hill.

López, G. F. (2004). "Dobles sentidos y connotaciones en el discurso administrativo". En: Ad-minister. Universidad EAFIT. Volumen (5): 11-21.

Mayo, E. (1977). Problemas sociales de una civilización industrial. Buenos Aires: Nueva Visión.

Robbins, S. (2004). Comportamiento organizacional. México: Prentice.

Simon, H. A. (1972). El comportamiento administrativo. Madrid: Aguilar.

Skinner, B. F. (1977). Sobre el conductismo. Barcelona: Fontanela.

Zapata, A. (2007). Cultura organizacional. Cali: Ediciones Universidad del Valle. 